\title{
HTLV-1 associated T cell lymphoma in South East Asia: Case report and family study
}

\author{
T L Wang, T C Liu, L Chan, Y K Kueh, S Y Wong
}

\begin{abstract}
Geographic clustering of human $T$ cell lymphoma/leukaemia virus type 1 (HTLV-1) infection is well recognised, particularly in south western Japan, parts of West and Central Africa, the south eastern United States and the Caribbean islands. ${ }^{1}$ Sporadic cases have been reported in many other parts of the world. The first case of HTLV-1 associated leukaemia/lymphoma (ATLL) in South East Asia is reported. Contact tracing showed a high incidence of carriers among the relatives.
\end{abstract}

\section{Case report}

A 52 year old Chinese woman was referred for evaluation of an abdominal mass and cervical lymphadenopathy. A cervical lymph node was subsequently biopsied. Sections showed the nodal architecture to be completely effaced by neoplastic lymphoid cells which exhibited a high mitotic rate and displayed bizarre shapes with pronounced nuclear variation and multilobation (fig A). Immunophenotyping using a panel of monoclonal antibodies applied to cryostat sections of fresh lymph node tissue showed surface expression of $\mathrm{CD} 2, \mathrm{CD} 8$,
HLA-DR and CD25 with weak positivity for CD4 and CD7 in the lymphoid cells (figs BD). The features were compatible with a high grade pleomorphic medium and large cell type, T-cell non-Hodgkin's lymphoma. ${ }^{2}$

Staging of her disease showed marrow infiltration by similar pleomorphic lymphoid cells and pronounced para-aortic lymphadenopathy. She was treated with combination chemotherapy (cyclophosphamide, doxorubicin, vincristine and prednisolone), but she died from progressive disease two months after diagnosis.

In view of the histological findings we tested her serum for the presence of antibodies to HTLV-1. An enzyme linked immunosorbent assay (ELISA) showed reactivity to HTLV-1 viral proteins and Western blot analysis (Diagnostic Biotechnology, Singapore) showed reactivity to both gag and env HTLV-1 proteins. She was seronegative for antibody to human immunodeficiency virus.

A high incidence of HTLV-1 infections among relatives of patients with HTLV-1 associated leukaemia/lymphoma can be expected. $^{3}$ We tested the sera of nine of her relatives. Four $(44 \%)$ of them had HTLV-1 infections by both enzyme linked immunosorbent assay and Western blot analysis (fig E).
Department of Medicine, National University Hospital, Lower Kent Ridge Road, Singapore 0511 T L Wang

Y K Kueh

\section{Department of}

Laboratory Medicine,

National University

Hospital

T C Liu

Department of

Pathology, National

University Hospital

$S$ Y Wong

Diagnostic

Biotechnology (Pte)

Ltd, Singapore

L Chan

Correspondence to:

Dr T L Wang

Accepted for publication 7 February 1991
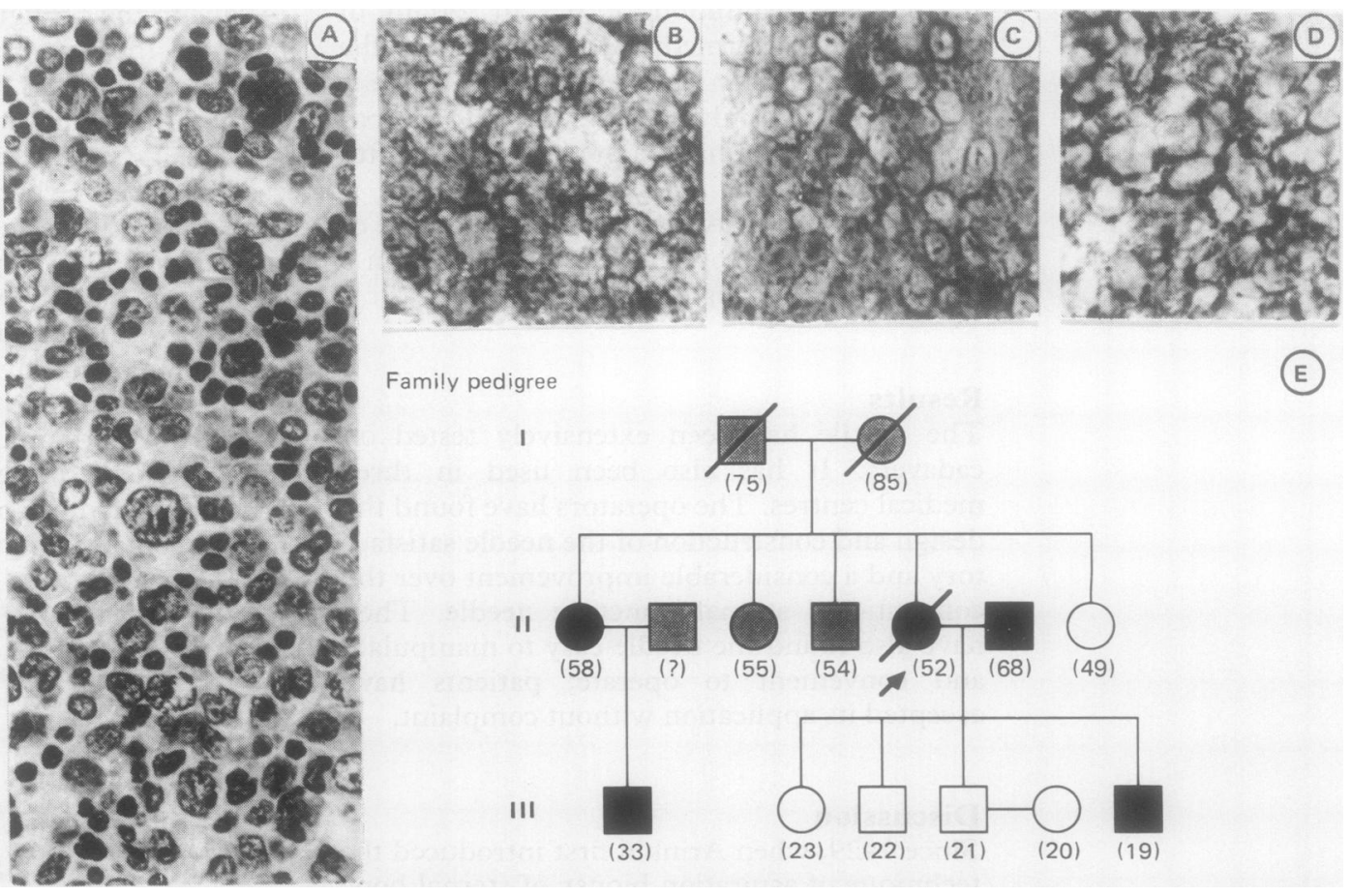

Family pedigree

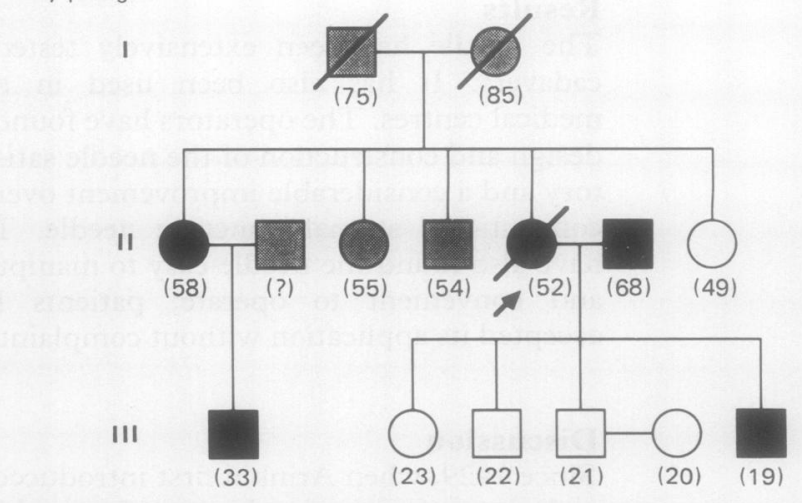

HTLV-1 associated ATLL: (A) bizarre lymphoid cells in the lymph node with nuclear irregularity (haematoxylin and eosin). (B) CD2; (C) HLA-DR; and (D) CD25-interleukin-2 receptors are positive on the surface of these lymphoid cells (immunoperoxidase/haematoxylin). (E) Family pedigree of the affected patient (arrowed). Shaded in black are those found to be HTLV-1 seropositive; shaded in white are those.found to be seronegative; and shaded in grey are those whose blood was not available for analysis. Numbers in parentheses refer to age in years. 
None of them had a history suggestive of tropical spastic paraparesis, the other known syndrome associated with HTLV-1 infection. Our patient was a second generation immigrant from south China. She had never travelled to HTLV-1 endemic areas, but had received a blood transfusion for post-partum haemorrhage during delivery of her first child.

\section{Comment}

HTLV-1 infections have not been widely documented in east Asia outside of Japan and Taiwan. The seroepidemiological pattern of this infection in South East Asia has not been well studied. A recent serological survey of 9689 healthy blood donors and pregnant women in Singapore showed an incidence of $0.03 \%$ (unpublished data). This is close to the $0.025 \%$ seroprevalence documented in blood donors in the United States. ${ }^{4}$

The fact that both vertical and horizontal transmission of this infection can readily occur is well illustrated by this case. This ease of transmission is of concern, especially when pockets of HTLV-1 infection have been documented in unusual areas. ${ }^{5}$ At the least, seroepidemiological trends must be monitored to ensure that the problem does not take on greater proportions.
The low seroprevalence of HTLV-1 in non-endemic areas is likely to preclude costeffective screening of blood donors. Patients with mature T-cell lymphoid malignancies, however, should be screened for infection. Contact tracing after identification of the index case is likely to be fruitful. The detection of infected patients, together with counselling on the modes of transmission, may help to prevent further transmission and protect national blood resources. We thank Dr T P Y in of the Specialist Centre in Penang for
his cooperation. This work was supported in part, by the his cooperation. This work was supported in part, by the
National University of Singapore Biological Sciences Research National University
Project RP 890383.

1 Levine PH, Blattner W, Clark J, et al. Geographic distribution of HTLV-1 and identification of a new high-risk population. Int $J$ Cancer 1988;42:7-12.

2 Suchi T, Lennert K, Tu LY, et al. Histopathology and immunohistochemistry of peripheral T cell lymphoma: a proposal for their classification. J Clin Pathol 1987;40: 995-1015.

3 Sakuma M, Takiguchi T, Konda S. HTLV-1 carriers in ATLL-nonendemic area with reference to blood transfusions. Nippon Ketsueki Gakkai Zasshi 1989;52:869-79.

4 Williams AE, Fang CT, Slamon D, et al. Seroprevalence and epidemiologic correlates of HTLV-1 infection in US blood donors. Science 1988;240:643-6.

5 Weber J. HTLV-1 infection in Britain. Official recognition of the need for surveillance is overdue. $\mathrm{Br} \mathrm{Med} \mathrm{J} \mathrm{1990;}$ 301:71-2.

\title{
Treatment of acute myeloid leukaemia in a renal allograft recipient: Implications of cyclosporin immunosuppressive treatment
}

\author{
R J G Cuthbert, N H Russell, P A E Jones, A G Morgan
}

Department of

Haematology, City

Hospital, Nottingham

NG5 1PB

R J G Cuthbert

N H Russel

P A E Jones

Department of Renal

Medicine

A G Morgan

Correspondence to:

Dr R J G Cuthbert

Accepted for publication

12 February 1991

\begin{abstract}
The clinical effects of cyclosporin were evaluated during cytotoxic treatment in a 61 year old man with acute myeloid leukaemia. He had required a renal transplant 18 months before presenting with acute myeloid leukaemia (FAB subtype M4). He had received cyclosporin $3.5-4.0 \mathrm{mg} / \mathrm{kg}$ daily to maintain a plasma cyclosporin concentration of 75-150 ng/ ml. Cyclosporin was continued during induction chemotherapy with daunorubicin, cytarabine, and 6-thioguanine (DAT). He had fever and oropharyngeal candidiasis that was unresponsive to anti-bacterial drugs but responsive to systemic amphotericin. Bone marrow examination 14 days after chemotherapy showed complete haematological remission. Subsequently he tolerated consolidation treatment with DAT with no
\end{abstract}

serious complications. Unfortunately he developed fatal septicaemia following a second consolidation with mitozantrone and cytarabine.

Inhibition of P-glycoprotein activity by cyclosporin may not significantly increase the toxicity of aggressive chemotherapeutic regimens, and as benefit may be achieved by this approach further clinical evaluation is justified.

In the management of malignant disease resistance to cytotoxic drugs often makes treatment unsuccessful. Interest has developed in studying the possible benefits of inhibiting the activity of P-glycoprotein to circumvent cytotoxic drug resistance. ${ }^{1} \mathrm{P}$-glycoprotein is a 170 kilodalton transmembrane glycoprotein which acts as an energy dependent pump, causing active efflux of structurally hetero- 